\title{
A Novel CPW Fed Dual-band Microstrip Antenna
}

\author{
Feng-rong $\mathrm{LI}^{1,{ }^{*}}$ and Ting WANG ${ }^{1}$ \\ ${ }^{1}$ National Key Laboratory for Mechatronical Engineering and Control, School of \\ Mechatronical Engineering, Beijing Institute of Technology, Beijing, China \\ *fengrongli09@126.com
}

Keywords: RF MEMS, Microstrip antenna, Dual-Band, CPW.

\begin{abstract}
In view of the low gain and single frequency operation of common microstrip antenna, a novel dual-band microstrip antenna which is fed by microstrip line is proposed in this paper. Using software HFSS to simulate and optimize it, the antenna can work at two bands which are $3.25-3.35 \mathrm{GHz}$ and $4.31-4.42 \mathrm{GHz}$. The total bandwidth is $0.21 \mathrm{GHz}$, while the gain is only $2.1 \mathrm{~dB}$.In order to further improve the gain and the bandwidth, a dual-band microstrip antenna with coplanar waveguide (CPW) fed is designed, the current distribution of the patch is changed by etching U-shaped and V-shaped slots. The return loss and the radiation efficiency are improved by adopting the CPW feeding structure. The simulation results show that the antenna can work at the two bands of $4.25-4.35 \mathrm{GHz}$ and $4.64-4.8 \mathrm{GHz}$ simultaneously. The maximum gain is $5.1 \mathrm{~dB}$. The performance parameters of the optimized antenna are improved compared with those of the original antenna. It can meet the application requirements of dual- band microstrip antenna.
\end{abstract}

\section{Introduction}

Due to the advantages such as small size, light weight, high reliability and easy conformal, the microstrip antenna has been widely concerned by domestic and foreign scholars[1]. The microstrip antenna is expected to be able to work in two or more discrete bands to improve the spectrum utilization, reduce the interference between the frequency spectrums and achieve more functions. The WLAN (wireless local area network), GPS (Global Positioning System) and other communication systems are all typical examples [2-3] to work in different frequency bands. There are some common methods to realize the multiband characteristic: the first one is that cutting slots in the patch [4-6].In this way, the surface current can form a meandering structure and then change the resonant frequency of the antenna. It is simple and convenient to achieve. The second way is loading components to realize the frequency reconfigurable antenna [7-9]. Although it can work in multiple bands by controlling the number of elements, the loss of the antenna is large and the radiation efficiency is low. The last method is that using the multi stacked patches, which has remarkable effect on realizing the multiband operation [10].However, the coupling between the antenna patches and the total thickness of the antenna is increased. In this paper, by etching U-shaped and V-shaped slots, a novel dual-band microstrip antenna with CPW fed is proposed.

\section{Structure Design and Simulation of Original Microstrip Antenna}

\section{Structure Design}

In order to realize the dual-band operation, a microstrip antenna with microstrip line fed is designed. The schematic diagram of the structure is shown in Fig. 1. The 
antenna consists of four parts, they are just as follows: radiation patch, microstrip feed line, dielectric substrate and ground plate. Four small rectangular patches are symmetrically loaded on the upper end of the patch, the dimensions are $4 * 3 \mathrm{~mm}^{2}$ and $4 * 3.5 \mathrm{~mm}^{2}$. By changing the antenna structure, the surface current distribution on the patch can be changed, which will further improve the impedance bandwidth and the gain. The parameters are shown in Table 1.

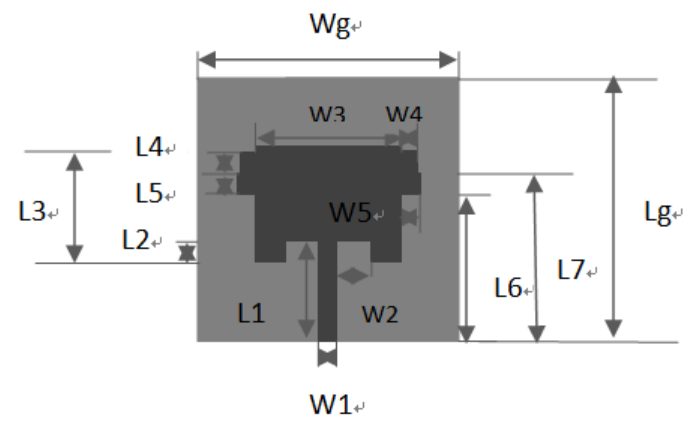

Fig. 1. Structure of antenna

Table 1. Size of antenna

\begin{tabular}{|l|l|l|l|l|l|l|l|}
\hline Parameter & W1 & L1 & W2 & L2 & W3 & L3 & L6 \\
\hline Unit(mm) & 3.5 & 20 & 6.5 & 4 & 28 & 22.39 & 29 \\
\hline Parameter & W4 & L4 & W5 & L5 & Wg & Lg & L7 \\
\hline Unit(mm) & 3.5 & 4 & 3 & 4 & 50 & 50 & 33 \\
\hline
\end{tabular}

The thickness of the antenna is $1.6 \mathrm{~mm}$ and the RF4 whose dielectric constant is 4.4 is used as the dielectric material. The equation for calculating the width of the radiation patch is as follow [11]:

$$
W=\frac{c}{2 f_{r}}\left(\frac{\xi_{r}+1}{2}\right)^{-1 / 2}
$$

In the Eq.1, the $C$ is the speed of light in vacuum, the $f_{r}$ is the resonant frequency and the $\varepsilon_{\mathrm{r}}$ is the dielectric constant of the substrate. From the Eq.1, we know that, after the dielectric constant is determined, the width of the microstrip antenna has an inverse relationship with resonant frequency.

The length of the patch is defined as $\lambda_{\mathrm{g}} / 2$, the $\lambda_{\mathrm{g}}$ is the guide wavelength in dielectric material. The equation is as follow:

$$
\lambda_{g}=\frac{c}{f_{r} \sqrt{\xi_{e}}}
$$

In the equation, the $\varepsilon_{\mathrm{e}}$ is the effective dielectric constant of the substrate materials. Due to the effect of the fringing field shortening, the actual length of $\mathrm{L}$ should subtract $2 \Delta \mathrm{L}$; the length of the patch edge is calculated as follow:

$$
\Delta L=0.412 h \frac{\left(\xi_{e}+0.3\right)(W / h+0.264)}{\left(\xi_{e}-0.258\right)(W / h+0.8)}
$$

The length of the microstrip antenna can be calculated as follow:

$$
L=0.5 \lambda_{g}-2 \Delta L
$$




\section{Simulation Results}

After using the software HFSS to simulate and optimize the antenna, the return losses and gain are shown in Fig. 2 and Fig. 3 respectively. It can be seen from the Figure2 that there are two resonant points which are $3.31 \mathrm{GHz}$ and $4.36 \mathrm{GHz}$. The total bandwidth is $0.21 \mathrm{GHz}$, while the return losses at the corresponding points are $-13.5 \mathrm{~dB}$ and $-21.6 \mathrm{~dB}$ and the maximum gain is only $2.1 \mathrm{~dB}$, which show that the radiation characteristics and gain of the antenna are still need to be improved.

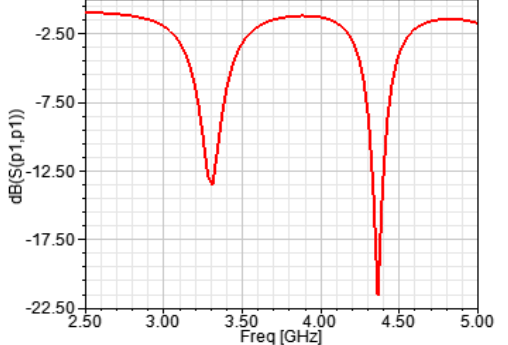

Fig. 2. Return loss

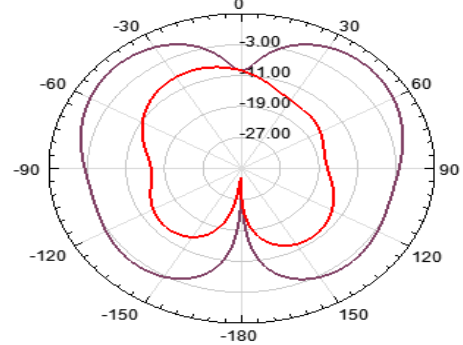

Fig. 3. Antenna gain

\section{Structure Design and Simulation of a Slotted Microstrip Antenna}

\section{Structure Design}

In order to further broaden the bandwidth, increase the gain and improve the return losses, a V shaped slot is etched on the patch. The schematic diagram of the structure is shown in Fig. 4. The size of the antenna is $50 * 50 * 1.6 \mathrm{~mm}^{3}$. The length of the three sides are $15 \mathrm{~mm}, 8.15 \mathrm{~mm}$ and $8.15 \mathrm{~mm}$ in turn.

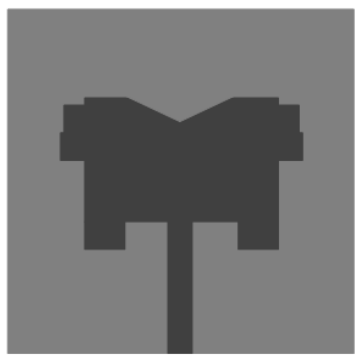

Fig. 4. Structure of antenna

\section{Simulation Results}

After using the software HFSS to simulate and optimize the antenna, the return losses and gain of the antenna are shown in Fig. 5 and Fig. 6 respectively. As we can see from the Fig. 5, there are two resonant points which are $3.35 \mathrm{GHz}$ and $4.42 \mathrm{GHz}$. The total bandwidth is $0.22 \mathrm{GHz}$. The return losses at the corresponding points are $-14.1 \mathrm{~dB}$ and $-22.8 \mathrm{~dB}$ and the maximum gain is $3.7 \mathrm{~dB}$.As a result, the gain, total bandwidth and return losses are all improved. But from figure of the return loss at $3.35 \mathrm{GHz}$, we know that the impedance matching can be better.

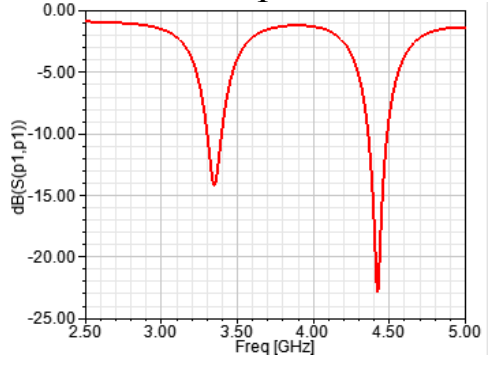

Fig. 5. Return loss

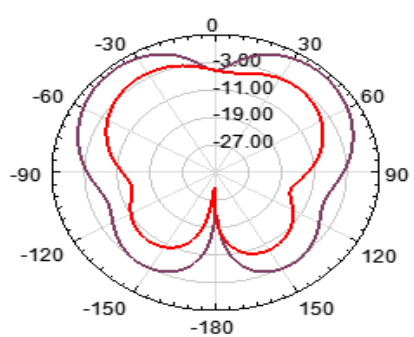

Fig. 6. Antenna gain 


\section{Structure Design and Simulation of a Slotted Microstrip Antenna with CPW Fed}

\section{Structure Design}

In order to further reduce the return loss and improve the impedance matching, on the basis of original structure, a U-shaped slot and CPW fed is provided for the antenna. As we know, the U-shaped slot can increase the length of the current, and the CPW not only plays a role of feeding but also acts as part of the antenna. By using the CPW fed, the size of the patch is increased and the loss of the antenna is reduced. The performance parameters of the antenna are improved significantly. The schematic diagram of the structure is shown in Fig. 7. The size of the antenna is still $50 * 50 * 1.6 \mathrm{~mm}^{3}$.The length, width and height of the U-shaped slot are $30 \mathrm{~mm}, 1.1 \mathrm{~mm}$ and $4 \mathrm{~mm}$. The gap between the ground plate and the microstrip line is $2.6 \mathrm{~mm}$, the height of the ground plate is $13 \mathrm{~mm}$.

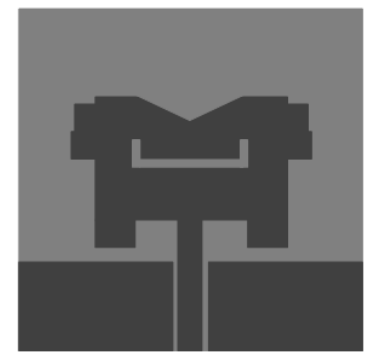

Fig. 7. Structure of antenna

\section{Simulation Results}

After using the software HFSS to simulate and optimize the antenna, the return losses and gain of the antenna are shown in Fig. 8 and Fig. 9. The antenna optimized can work at the points of $4.29 \mathrm{GHz}$ and $4.72 \mathrm{GH}$, the return losses at the corresponding points are $-22.3 \mathrm{~dB}$ and $-31 \mathrm{~dB}$ accordingly. The total bandwidth is $0.27 \mathrm{GHz}$ and the maximum gain is $5.1 \mathrm{~dB}$ with good radiation characteristics.

In a word, by using the CPW fed and etching the U-shaped and V-shaped slots, the antenna has good radiation characteristics at each resonant point, the gain is improved significantly and the bandwidth is further broadened.

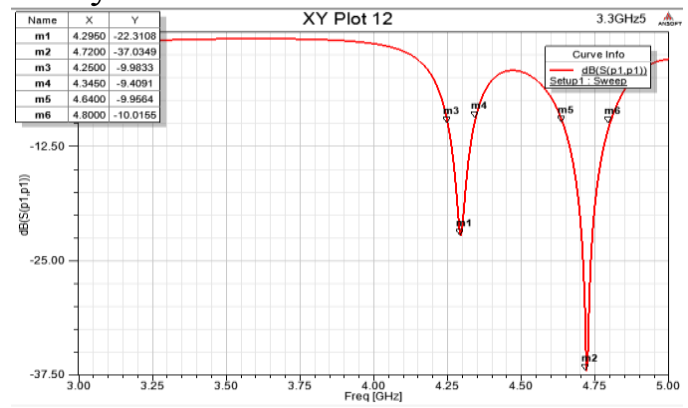

Fig. 8. Return loss

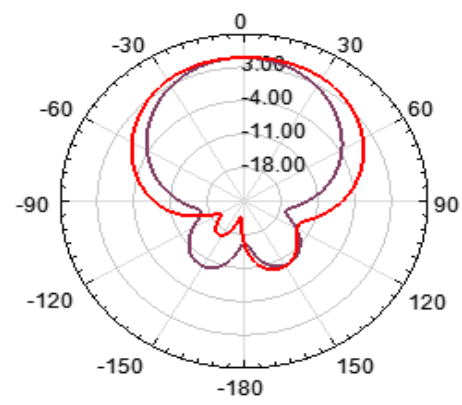

Fig. 9. Antenna gain 


\section{Conclusions}

To solve the problems of low gain and single frequency operation of the antenna, a novel dual-band microstrip antenna with CPW fed is proposed in the paper. By etching the U-shaped and V-shaped slots, the antenna can work at the bands of $4.25-4.35 \mathrm{GHz}$ and $4.64-4.8 \mathrm{GHz}$ at the same time. The return losses are $-22.3 \mathrm{~dB}$ and $-31 \mathrm{~dB}$ accordingly and the maximum gain of the antenna is $5.1 \mathrm{~dB}$. The antenna designed can meet the application requirements of antenna in both civil and military fields.

\section{References}

[1] Rabies G M.RF MEMS: Theory, Design and Technology [M]. New Jersey: John Willey and Sons, 2003.

[2] Yanhang Pu. Research on dual-band microstrip antenna [D]. Nanjing: Nanjing University of Science and Technology, 2008.,pp.3-7.

[3] Yu Jia, Jianxia Liu, Dongmei Cai, Shijun Yan. Double-1-shaped dual-band microstrip antenna design for WLAN/WiMAX applications [J]. Electronic Components and Materials, 2014, 33(12),pp.52-56.

[4] Cherukuri Keerthi, Jasti.Raja Sekhar. Reconfigurable Multi- Band Antenna Using RF MEMS [J]. International Journal of Computer Science Engineering and Technolog, 2014, 4(5),pp. 176-178.

[5] T. H. Kim and D. C. Park, "Compact dual-band antenna with double L-slits for WLAN operations," IEEE Antennas Wireless Propag. 2005,pp.249-252.

[6] Dongsheng, Qi, Binhong, Li, Haitao, Liu, YaBin, Zhang. Slot-loaded H-shaped dual-frequency antenna [J]. Chinese Journal of Radio Science, 2004, 19(1),pp.95-98.

[7] Libo, Chen, YaNan, Xie, Zimiao, Tan, Jiajia, Wang. Frequency reconfigurable UWB antenna [J]. Electronic Measurment Technology, 2015, 38(1),pp.7-11.

[8] Jing Liang, H Y David Yang. Reconfigurable patch antenna on a tunable EBG substrate[C].IEEE Antennas on Propagation Symposium, Japan Niigata,2007,pp.5219 $-5222$.

[9] Symeon Nikolaou, Ramanan Bairavasubramanian. Pattern and frequency reconfigurable annular slot antenna using PIN diodes[J]. IEEE Transactions on Antennas andPropagation, 2006, 54(2),pp.439-447.

[10] Chen Wang. Research on novel multiband and compact antenna based on composite right/left -handed transmission lines structures [D]. Guangzhou: South China university of technology, 2012,pp.75-90.

[11] Mingyang Li, Min Liu, Fang Yang.HFSS antenna design [M].Beijing: House of Electronics Industry, 2007,pp.99-101. 